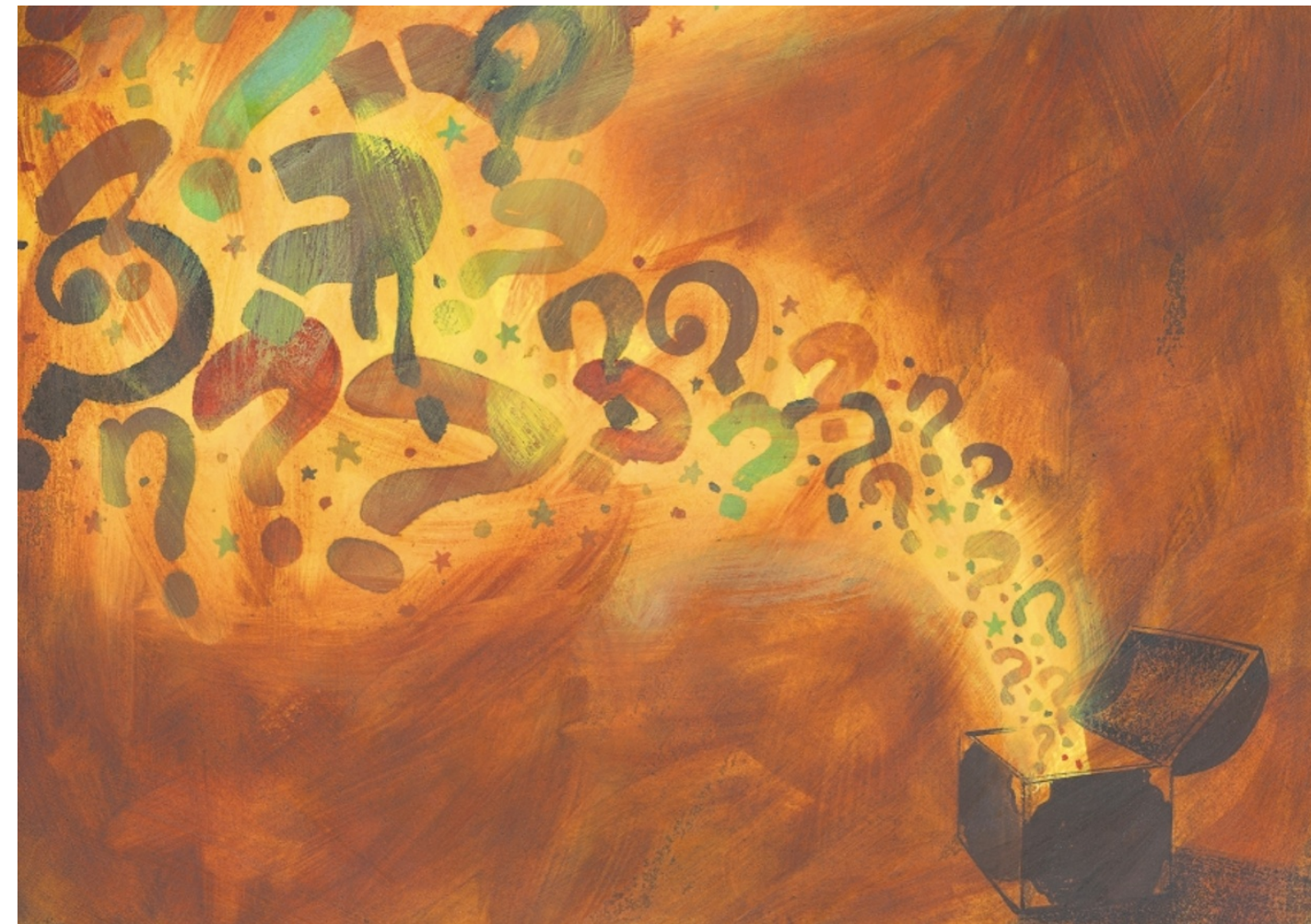

HORMONE SIGNALLING

\section{A mystery unfolds}

The bigger a molecular complex, the more cumbersome and time consuming it will be to put together and break apart. Yet large hormone-receptor complexes can respond very rapidly to changes in hormone levels, and can turn transcription on or off within a matter of minutes. How they do this has been a puzzle, but Brian Freeman and Keith Yamamoto now provide a possible answer - these complexes are rapidly dismantled by molecular chaperones.

The authors studied a group of receptors known as the intracellular receptors (IRs). These form hormone-receptor complexes that bind selectively to sites on the DNA ('hormone response elements'; HREs) and trigger the formation of large transcriptional regulatory complexes. IRs do not seem to be degraded when the hormone is withdrawn, so the authors wondered whether they might instead be reversibly inactivated by chaperones - as these can alter protein conformation, they might serve to trigger a molecular switch.

Reporting in Science, Freeman and Yamamoto provide evidence that chaperones are indeed involved. They studied the effects of a chaperone called p 23 on a regulatory complex that was formed by interaction of hormone-bound thyroid hormone receptor (TR) with the thyroid response element (TRE).

The authors incubated p 23 with the TR-TRE complex, then added a HeLa nuclear extract as a source of transcription factors. The result was reduced transcriptional activation by the receptor in the presence of $\mathrm{p} 23$ compared with controls that lacked the chaperone. They also preformed the transcriptional regulatory complexes by preincubating the TR-TRE complexes with HeLa extract. Again, transcription was blocked in the presence of the chaperone, which indicates that p23 can disassemble TR-TRE-containing regulatory complexes in vitro.

To confirm these results in vivo, Freeman and Yamamoto developed a way to selectively increase the local concentration of the chaperone close to the HRE of choice. They studied three chaperones - p23, Hsp90 and Hsp70. Expression of p23 reduced transcriptional activation by the TR and the glucocorticoid receptor (GR) by 100 -fold and 35 -fold, respectively. Hsp90 had more modest effects on the GR only, and Hsp70 did not affect transcription by either receptor. Significantly, basal levels of transcription were unaffected, presumably because these chaperones act specifically on certain target proteins.

Given these results, the authors next asked whether components of these molecular chaperone complexes could be detected at functional HREs in vivo. They used chromatin immunoprecipitation to show that p23 and Hsp90 (but not Hsp70) indeed localized to HREs in a hormone-dependent manner.

A final finding - with startling implications - is that p23 and Hsp90 can influence the activities of belong to the IR family. The authors showed that both proteins can reduce transcriptional activity by NF- $\kappa B$ (in response to tumour-necrosis factor- $\alpha$ ), and that p23 also affects AP-1 (in response to phorbol 12myristate 13-acetate). This indicates that chaperones might act more widely, and could provide a general mechanism by which to disassemble transcriptional regulatory complexes.

Alison Mitchell

(2) References and links

ORIGINAL RESEARCH PAPER Freeman, B. C. \& Yamamoto, K. R. Disassembly of transcriptional regulatory complexes by molecular chaperones. Science 296, 2232-2235 (2002) WEB SITE

Keith Yamamoto's laboratory: www.ucsf.edu/krylab transcriptional regulatory complexes that do not

\section{IN THE NEWS}

\section{Do-it-yourself virus}

"Scientists... have, for the first time, fabricated a working copy of a dangerous disease virus from scratch in the lab", reported The Guardian (12 July 2002).

Eckhard Wimmer and colleagues at the State University of New York spent three years

constructing de novo infective poliovirus using only the publicly available viral sequence and mailorder DNA

oligonucleotides. They joined plus and minusstrand oligonucleotides into full-length poliovirus cDNA and used this to transcribe viral RNA. This was translated into protein, which, after replication, was found to infect mouse cells.

The study, published online in Science, which was carried out "to prove that it could be done", is being seen as a landmark paper in virology.

"Understanding the process of viral DNA production is

key to identifying new ways to kill viruses and

understand how viruses could change and escape from vaccines", said The Defense Advanced Research Projects Agency, which provided funding for the research.

But scientists are worried about the serious ramifications of the study. "To purposely make a synthetic human pathogen is irresponsible", Craig Venter told The New York Times (12 July 2002).

Others, including the authors, are worried because genetic sequences such as Ebola, smallpox and HIV are freely available on the Internet. This, coupled with the relative ease with which Wimmer's group made an infectious virus, raises concerns over whether the technique could be exploited by terrorists to make biological weapons without obtaining a natural virus.

Katrin Bussell 